\title{
Dynamique non-linéaire des vagues en zone de surf interne
}

\author{
Philippe Bonneton
}

Chargé de Recherche du CNRS au Département de Géologie et d'Océanographie, UMR CNRS 5805, Université de Bordeaux I, France. Tél :05579629 65, p.bonneton@geocean.u-bordeaux.fr

\section{Résumé}

Nous présentons dans cet article un modèle mathématique intégré suivant la verticale qui permet de calculer la transformation non-linéaire des vagues en zone de surf interne. Nous analysons tout d'abord les processus de distorsion et de dissipation des vagues à partir de la théorie hyperbolique des ondes de choc. Nous présentons ensuite une comparaison entre les résultats expérimentaux de Cox (1995) et nos simulations numériques, qui montre la capacité de notre modèle à reproduire la dynamique des vagues en zone de surf interne.

\begin{abstract}
In this paper we present a depth-integrated mathematical model for computing the non-linear transformation of waves in the inner surf zone. First, we analyse wave distortion and dissipation processes from the hyperbolic theory for shock wave. Then, we present a comparison between experimental results by Cox (1995) and our numerical simulations, which shows the ability of our numerical model to describe the wave dynamics in the inner surf zone.
\end{abstract}

\section{Introduction}

La houle et les courants qu'elle induit jouent un rôle essentiel dans la formation des structures sédimentaires côtières. Une connaissance précise des phénomènes associés aux vagues est primordiale pour la compréhension des différentes formes de barres (multiples ou pas, localisation par rapport à la zone de surf, longshore, obliques ou en croissant...). La houle à l'approche de la côte suit l'évolution schématique suivante : elle se raidit en même temps que la profondeur d'eau diminue ("zone de levée"), jusqu'au déferlement associé à des mouvements turbulents intenses ("zone de déferlement"), puis les vagues se réorganisent sous la forme de ressauts hydrauliques propagatifs ("zone de surf interne" (ZSI)), qui atteignent la plage en générant des jets de rive ("zone de swash"). Si les phénomènes de réfraction et diffraction dans la zone de shoaling sont maintenant bien pris en compte dans les modèles basés sur les équations de Berkhoff ou de Boussinesq, en revanche la compréhension des processus hydrodynamiques après le déferlement reste un domaine de recherche ouvert. 
Nous présentons dans cet article un modèle mathématique intégré suivant la verticale (section 2) qui permet de décrire la propagation et la dissipation des vagues en ZSI. Nous proposons ensuite à la section 3 une analyse théorique, à partir de ce modèle, de la distorsion des vagues en ZSI. Pour terminer (section 4) nous présentons une validation de ce modèle à partir de l'expérience de laboratoire de Cox (1995).

\section{Modèle mathématique}

\subsection{Equations du mouvement intégrées suivant la verticale}

Dans la ZSI la longueur d'onde des vagues est grande devant la profondeur d'eau. Il est alors classique d'essayer de réduire la complexité du problème en intégrant suivant la verticale les équations du mouvement. Le déferlement est généralement de type déversant dans la ZSI et l'on peut donc considérer que les hypothèses sur lesquelles repose la condition cinématique à l'interface (surface libre continue et monovaluée) sont justifiées. L'intégration suivant la verticale des équations de Navier Stokes conduit alors au résultat suivant :

$$
\begin{aligned}
\frac{\partial h}{\partial t}+\frac{\partial h u}{\partial x} & =0 \\
\rho \frac{\partial h u}{\partial t}+\rho \frac{\partial}{\partial x}\left(h u^{2}+\frac{1}{2} g h^{2}\right) & =\rho g h \frac{\partial d}{\partial x}+N_{H y}+V_{v e r}+D_{i f f}-\tau_{b}
\end{aligned}
$$

où, $h(x, t)$ est la hauteur d'eau, $d(x)$ la profondeur d'eau au repos, $\zeta(x, t)$ la cote de la surface libre $(\zeta(x, t)=h-d), \rho$ la densité de l'eau, $u$ la vitesse moyennée suivant la verticale $\left(u=\frac{1}{h} \int_{-d}^{\zeta} v_{1} d z, v_{1}\right.$ composante de la vitesse suivant $x), \tau_{b}$ la tension de cisaillement au fond et

$$
\begin{aligned}
N_{H y} & =\frac{\partial}{\partial x}\left(\int_{-d}^{\zeta}-p d z\right)+p(-d) \frac{\partial d}{\partial x} \\
V_{v e r} & =-\rho \frac{\partial}{\partial x}\left(\int_{-d}^{\zeta} \tilde{u}^{2} d z\right) \\
D_{i f f} & =\frac{\partial}{\partial x}\left(\int_{-d}^{\zeta} \tau_{x x}^{v} d z\right)
\end{aligned}
$$

$P$ la pression, $p=P-\rho g(\zeta-z)$ la pression non-hydrostatique, $\tilde{u}=v_{1}-u$ et $\tau_{x x}^{v}$ la composante suivant $x$ du tenseur des contraintes visqueuses. Dans l'équation de conservation de la quantité de mouvement horizontale (2), $N_{H y}$ correspond à la contribution des effets non-hydrostatiques, $V_{v e r}$ à la variabilité verticale du champ de vitesse et $D_{i f f}$ à la diffusion de la quantité de mouvement. Il est important de noter qu'à ce stade de développement la seule hypothèse qui a été émise pour obtenir ce système d'équations concerne l'utilisation de la condition cinématique à la surface libre.

On observe que dans la ZSI les variables hydrodynamiques évoluent de façon régulière, excepté très localement au niveau de fronts d'ondes. L'objectif dans cette section est d'établir un modèle mathématique qui décrive de façon 
précise la propagation et la distorsion des vagues sans tenir compte de façon détaillée de la structure des fronts d'onde. Pour cela nous nous inspirons de la démarche développée dans le domaine de la mécanique des fluides compressibles pour l'études des ondes de choc (cf. Lax (1957)).

Des expériences de laboratoire comme celles de Cox (1995) et Ting et Kirby (1996), ainsi que les simulations numériques diphasiques de Lin et Liu (1998) ont permis de montrer que dans la ZSI, excepté au niveau des fronts d'onde, le champ de vitesse des vagues déferlées variait peu suivant la verticale et que la pression était proche de la pression hydrostatique. Ces observations nous conduisent à négliger $N_{H y}$ et $V_{v e r}$ dans l'équation 2. Dans la ZSI les termes visqueux agissent principalement au niveau des fronts d'onde, où sont localisés de très forts gradients de vitesse. Nous allons montrer par la suite qu'il est possible de négliger le terme de diffusion $D_{i f f}$ en introduisant le concept de choc pour décrire les fronts d'onde. On obtient alors comme modèle mathématique non visqueux le système d'équations suivant:

$$
\begin{aligned}
\frac{\partial h}{\partial t}+\frac{\partial h u}{\partial x} & =0 \\
\frac{\partial h u}{\partial t}+\frac{\partial}{\partial x}\left(h u^{2}+\frac{1}{2} g h^{2}\right) & =g h \frac{\partial d}{\partial x}-\frac{\tau_{b}}{\rho}
\end{aligned}
$$

où nous modélisons la tension de cisaillement au fond $\tau_{b}$ par $\tau_{b}=\frac{1}{2} \rho f|u| u(f$ est le coefficient de friction). Ce système correspond aux équations de Saint Venant avec friction.

\subsection{Chocs et dissipation}

Les équations (3) et (4) forment un système non-linéaire strictement hyperbolique (pour $h>0$ ) avec termes sources. L'absence de diffusion de la quantité de mouvement dans l'équation (4) conduit à la formation de discontinuités dans les solutions. Ces discontinuités (ou chocs) correspondent à la représentation mathématique des fronts d'onde. Il est alors nécessaire d'étendre les solutions du système (3) et (4) en utilisant le concept de solution faible (cf. Whitham (1974)). Les solutions faibles vérifient les équations (3) et (4), là où elles sont continues et les conditions de saut au niveau des chocs :

$$
\begin{aligned}
-U[h]+[h u] & =0 \\
-U[h u]+\left[h u^{2}+\frac{1}{2} g h^{2}\right] & =0
\end{aligned}
$$

Les crochets indiquent le saut des quantités et $U$ est la vitesse du choc. Il existe une indétermination concernant cette solution faible, qui ne peut être levée qu'en analysant l'évolution de l'énergie à travers un choc. En effet, pour qu'une solution faible soit physiquement admissible il est nécessaire qu'elle vérifie une condition d'entropie. Pour les équations de Saint Venant l'entropie "mathématique" correspond à l'énergie $\mathcal{E}=\frac{1}{2} \rho\left(h u^{2}+g(h-d)^{2}\right)$. La condition d'entropie en niveau d'un choc s'écrit :

$$
-U[\mathcal{E}]+[\mathcal{F}]<0
$$


où, $\mathcal{F}=\rho h u\left(\frac{1}{2} u^{2}+g(h-d)\right)$. Ceci exprime physiquement le fait que les particules de fluide perdent de l'énergie en traversant le choc. A partir des conditions de saut (5) et (6) on peut déterminer la dissipation d'énergie $\mathcal{D}=$ $-[\mathcal{F}]+U[\mathcal{E}]$ à travers un choc (cf. Bonneton $(2001))$ :

$$
\mathcal{D}=\frac{\rho g}{4}\left(h_{2}-h_{1}\right)^{3}\left(\frac{g\left(h_{2}+h_{1}\right)}{2 h_{1} h_{2}}\right)^{\frac{1}{2}}
$$

où $h_{1}$ et $h_{2}$ sont les hauteurs d'eau à l'avant et à l'arrière du choc.

Les équations de Saint Venant inviscides, associées au concept de solution faible, permettent donc de décrire la dissipation d'énergie au niveau des fronts d'onde. Bonneton (2001) a montré que ce modèle mathématique donnait une bonne description de la dissipation des vagues en ZSI. Il est important de noter que la dissipation donnée par l'équation (7) s'applique à tous les chocs et pas seulement au cas particulier du ressaut hydraulique. Or depuis les travaux de Le Méhauté (1962), il est classique dans le domaine du Génie Côtier d'estimer la dissipation dans les vagues déferlées en faisant une analogie entre ces fronts d'onde et le ressaut hydraulique propagatif. Les désaccords qui ont été observés entre les mesures et les modèles paramétriques basés sur cette analogie (cf. Battjes et Janssen (1978)) ont conduit un certain nombre d'auteurs à appliquer des facteurs correctifs à l'équation (7). Des calculs numériques (Kobayashi et coll. (1989), Cox (1995) et Bonneton et Dupuis (2001)) et théoriques (Bonneton (2001)) appliqués à la propagation des vagues en ZSI nous conduisent à penser que la dissipation donnée par l'équation (7) est en bon accord avec la réalité physique. Les facteurs correctifs appliqués à cette équation dans les modèles paramétriques servent seulement à compenser une mauvaise estimation des flux d'énergie dans ces modèles.

\section{Analyse de la distorsion des vagues}

Dans ce chapitre nous allons décrire les mécanismes non-linéaires qui contrôlent la distorsion des vagues en ZSI et qui conduisent à la forme caractéristique d'ondes en dents de scie. Pour simplifier le problème nous allons considérer une onde simple se propageant sans frottement sur un fond plat $\left(d(x)=d_{0}\right)$. Bonneton (2001) a montré que dans ce cas les équations de Saint Venant pouvaient se réduire à une équation de Burger. Dans le référentiel $\mathcal{R}_{0}$ se déplaçant à la vitesse $c_{0}=\left(g d_{0}\right)^{1 / 2}$, cette équation non-linéaire s'écrit :

$$
\frac{\partial u}{\partial t}+\frac{3}{2} u \frac{\partial u}{\partial x}=0
$$

avec en présence de choc la condition de saut : $U=\frac{3}{4}\left(u_{1}+u_{2}\right)$, où $u_{1}$ et $u_{2}$ sont les vitesses à l'avant et à l'arrière du choc. On peut obtenir l'élévation de la surface libre $\zeta$ en fonction de la vitesse à partir de la relation $\frac{\zeta}{d_{0}}=\frac{u}{c_{0}}\left(1+\frac{u}{4 c_{0}}\right)$. (On peut montrer que dans la ZSI $\frac{\zeta}{d_{0}} \simeq \frac{u}{c_{0}}$. L'analyse théorique des ondes que nous allons présenter à partir de champ de vitesse peut donc se transposer de façon directe à $\zeta$.) 


\section{VII $I^{e ̀ m e s}$ Journées Nationales Génie Civil - Génie Côtier, Anglet, France, 15-17 Mai 2002}
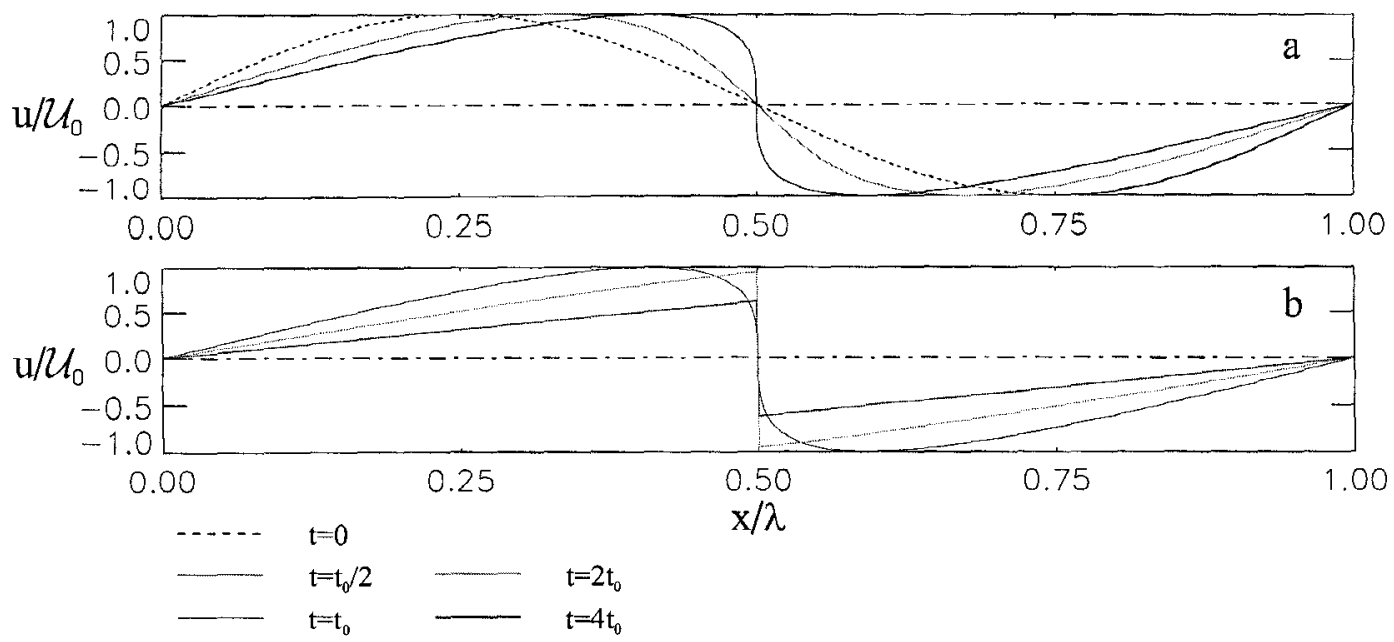

FIG. 1: Solutions analytiques de la distorsion d'une onde périodique sur fond plat. a, avant la formation des chocs; $b$, après la formation des chocs.

Fig. 1 : Analytical solutions of the distortion of a periodic wave on a flat bottom. a, before shock formation; b, after shock formation.

L'équation (8) signifie que la vitesse $u$ est constante le long des droites caractéristiques $\frac{d x}{d t}=c_{p}$, où $c_{p}=\frac{3}{2} u$ est la vitesse de propagation. Cette vitesse étant proportionnelle à $u$, la crête des ondes va se propager plus rapidement que les creux, ce qui conduit au raidissement des ondes et à la formation de chocs. Ces derniers vont se former aux points $x_{d}$ tels que la dérivée $\frac{\partial}{\partial x}\left(c_{p}(x, 0)\right)$ est négative et sa valeur absolue maximale et se produisent à l'instant $t=$ $\left.-1 / \frac{\partial c_{p}}{\partial x}\right)_{x_{d}}$.

Pour illustrer ce phénomène nous allons considérer une perturbation de vitesse initiale sinusoïdale de longueur d'onde $\lambda: u(x, t=0)=\mathcal{U}_{0} \sin (2 \pi x / \lambda)$. La vitesse de propagation $c_{p}$ étant proportionnelle à $u$, le champ d'onde va se distordre tout en préservant sa symétrie (cf. figure 1a). Les chocs se produisent en $\frac{x_{d}}{\lambda}=\frac{1}{2}+n(n \in N)$, à l'instant $t_{0}=\frac{\lambda}{3 \pi \mathcal{U}_{0}}$ et sont fixes dans le référentiel $\mathcal{R}_{0}$. On observe sur la figure $1 \mathrm{~b}$ que très rapidement après la formation des chocs, l'onde prend une forme en "dents de scie". Cette forme, qui est une des principales caractéristiques des vagues observées en ZSI (cf. figure 2), est donc le résultat des effets conjugués de la distorsion non-linéaire des vagues et de la dissipation de l'énergie au niveau des fronts d'onde.

Bonneton (2001), en utilisant cette théorie pour estimer les flux d'énergie et la dissipation en ZSI sur une plage à pente constante $\beta$ (faible), a pu déterminer analytiquement l'évolution de la hauteur des vagues $H$ en fonction de la profondeur $d(x)$ et du nombre sans dimension $\sigma=\frac{2 H_{0}}{\beta c_{0} T}$ :

$$
\frac{H}{H_{0}}=\left(\sigma\left(\frac{d}{d_{0}}\right)^{-1 / 2}+(1-\sigma)\left(\frac{d}{d_{0}}\right)^{1 / 4}\right)^{-1}
$$

où $H_{0}$ et $d_{0}$ correspondent à la hauteur des vagues et à la profondeur au début 


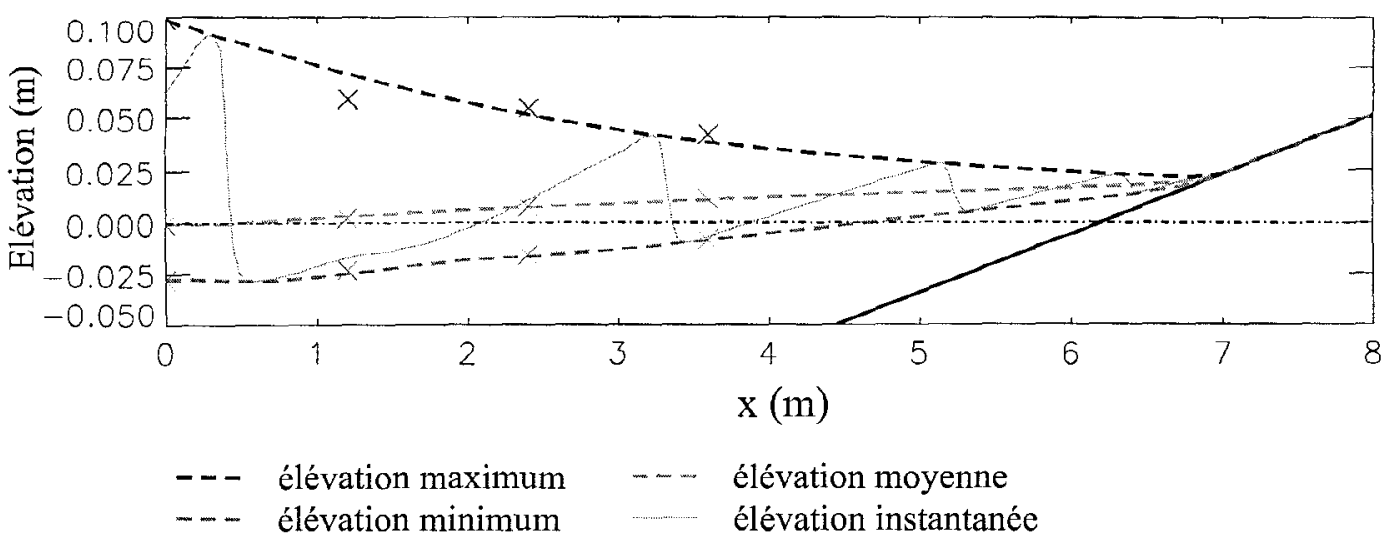

FIG. 2: Evolution spatiale de l'élévation des vagues. Comparaison entre le modèle numérique et les expériences de Cox (1995), (x).

Fig. 2 : Spatial evolution of wave elevation. Comparison between the numerical model and experiments by Cox (1995), (x).

de la zone de surf interne et $T$ est la période des vagues.

\section{Modélisation numérique}

Le modèle théorique de la section précédente nous a permis d'expliquer le phénomène non-linéaire de distorsion des vagues en ZSI. Cependant, pour calculer précisément les champs de vitesse et d'élévation de la surface libre il est nécessaire d'utiliser une résolution numérique des équations de Saint Venant, en particulier pour des situations complexes (houles et bathymétries irrégulières). Pour résoudre ce système d'équations non-linéaire hyperbolique nous avons développé un schéma numérique MacCormack TVD, qui donne une bonne description de la distorsion des vagues sans présenter d'oscillations numériques parasites au niveau des fronts d'onde (cf. Vincent, Bonneton et Caltagirone (2001)).

Nous présentons dans cette section une application de ce modèle à la propagation d'une houle régulière se propageant sur un fond de pente constante $\beta=1 / 35$, qui correspond à l'expérience de laboratoire réalisée par Cox (1995). Une houle de période $T=2.2 \mathrm{~s}$ et d'amplitude $H=13.22 \mathrm{~cm}$ est générée dans un canal de $33 \mathrm{~m}$ de long et déferle sous la forme d'un déferlement déversant. La condition limite de notre modèle numérique est donnée par les mesures de hauteur d'eau en un point situé au début de la ZSI ( $x=0$, sur nos figures). Nos calculs ont été réalisés avec une résolution spatiale $\Delta x=4 \mathrm{~cm}$, un pas de temps $\Delta t=0.01 \mathrm{~s}$ et un coefficient de friction $f=0.015$. Après une période de calcul de $100 T$ permettant d'évacuer les ondes transitoires nous avons réalisé une simulation sur une durée de $50 T$.

La figure 2 présente l'évolution spatiale de l'élévation des vagues dans la ZSI. On observe un très bon accord entre les valeurs calculées (élévations maximum, minimum et moyenne) et les valeurs mesurées. En particulier le modèle reproduit bien le phénomène de surélévation du plan d'eau ("set-up"). 

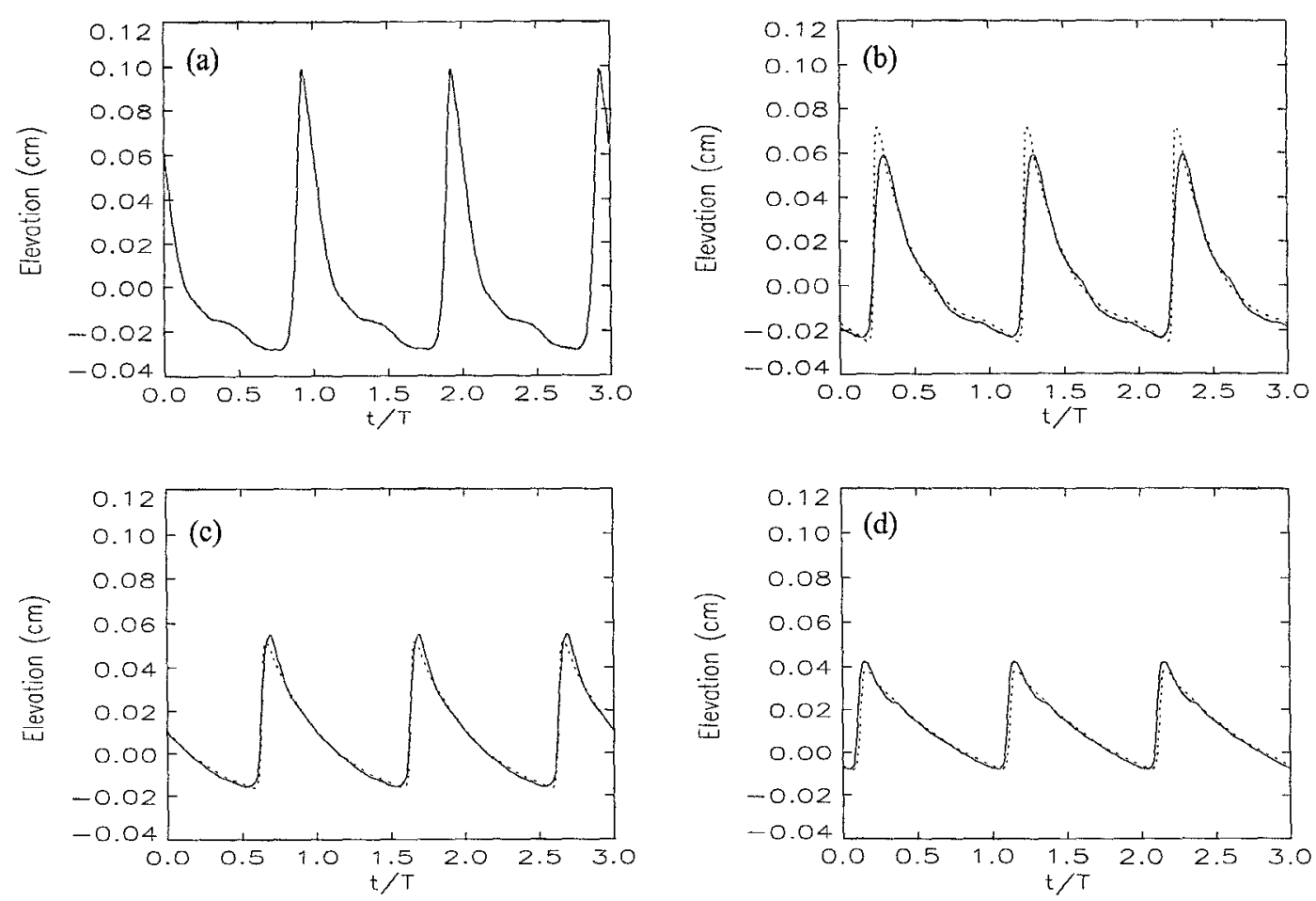

Fig. 3: Evolution temporelle de l'élévation des vagues en différents points de la ZSI. Comparaison entre le modèle numérique $(---)$ et les expériences de $\operatorname{Cox}(1995)$ (_ _ ) . a, $x=0 \mathrm{~m} ; \mathrm{b}, x=1.2 \mathrm{~m} ; \mathrm{c}, x=2.4 \mathrm{~m} ; \mathrm{d}, x=3.6 \mathrm{~m}$.

Fig. 3: Time evolution of wave elevation at different locations in the inner surf zone. Comparison between the numerical model (- - ) and experiments by $\operatorname{Cox}(1995)$ (__ ). $a, x=0 \mathrm{~m} ; b, x=1.2 \mathrm{~m} ; c, x=2.4 \mathrm{~m} ; d, x=3.6 \mathrm{~m}$.

Le champ de vague instantané que l'on peut observer sur la figure 2 illustre la forme caractéristique en dents de scie des vagues en ZSI. Cette figure montre aussi que pour des vagues régulières sur une plage de faible pente la zone de swash est pratiquement inexistante. Cette zone est présente lorsque la houle est irrégulière et est associée à des ondes infragravitaires. Une confrontation entre l'évolution temporelle de l'élévation mesurée et calculée en plusieurs points est présentée sur la figure 3. On observe un très bon accord, aussi bien sur la phase que l'amplitude des signaux. L'accord sur la décroissance de l'amplitude des vagues confirme la validité physique de notre modélisation de la dissipation.

\section{Conclusions et perspectives}

Nous avons présenté dans cet article un modèle mathématique intégré suivant la verticale qui permet de calculer la transformation non-linéaire des vagues en ZSI. Nous avons montré sa capacité à reproduire la distorsion des vagues ainsi que leur dissipation. Notre objectif est maintenant d'étendre le domaine d'application de notre modèle (de la zone de levée à la zone de swash) en développant, en collaboration avec T. Colin (Laboratoire de Mathématiques 
Appliquées de Bordeaux), une modélisation de type Boussinesq, qui permette en particulier de bien reproduire la dynamique non-linéaire et très faiblement dispersive des fronts d'onde en ZSI. Parallèlement à ce projet, nous allons étudier en collaboration avec J-P. Caltagirone (Laboratoire MASTER) les limites de validité des modèles intégrés suivant la verticale et leurs possibles améliorations, à partir de simulations numériques diphasiques du déferlement déversant.

Remerciements : Ces travaux ont été soutenus par deux programmes nationaux de l'INSU : le PNEC (Programme National d'Environnement Côtier) et le PATOM (Programme ATmosphère Océan à Multi-échelles). Nous remercions Daniel Cox pour avoir mis à notre disposition les données expérimentales issues de sa thèse.

\section{Références}

[1] Battjes, J.A. and Janssen, J.P.F.M. 1978 Energy loss and setup due to breaking of random waves. In Proc. Int. Conf. on Coastal Eng., ASCE, 569587.

[2] Bonneton, P. 2001 A note on wave propagation in the inner surf zone, C.R. Acad. Sci. Paris, 329, Série II b, 27-33.

[3] Bonneton, P. and Dupuis 2001 Transformation of irregular waves in the inner surf zone. Proc. 27th Int. Conf. on Coastal Eng, 1, 745-754.

[4] Cox, D.T. 1995 Experimental and numerical modelling of surf zone hydrodynamics, Ph.D. dissertation, Univ. of Delaware, Newark.

[5] Kobayashi, N., DeSilva, G.S. and Watson, K.D. 1989 Wave transformation and swash oscillation on gentle and steep slopes. J. Geophys. Res. 94 (C1), 951-966.

[6] Lax, P.D. 1957 Hyperbolic systems of conservation laws. Comm. Pure Appl. Math. 10, 537-566.

[7] Le Méhauté, B. 1962 On the non-saturated breaker theory and the wave run-up. Proc. 8th Coastal Engineering Conf. 77-92.

[8] Lin, P. and Liu, P. L.-F.1998 A numerical study of breaking waves in the surf zone, J. Fluid Mech., 359, 239-264.

[9] Ting, F.C.K. and Kirby, J.T. 1996 Dynamics of surf-zone turbulence in a spilling breaker. Coastal Eng., 27, 131-160.

[10] Vincent, S., Bonneton, P. and Caltagirone, J-P. 2001 Numerical modelling of bore propagation and run-up on sloping beaches using a MacCormack TVD scheme, J. of Hydr. Research, 39, 41-49.

[11] Whitham, G.B. 1974 Linear and nonlinear Waves. Wiley-Interscience Publication. 